\title{
Microscopic observation drug susceptibility assay for the diagnosis of TB and MDR-TB in HIV-infected patients: a systematic review and meta-analysis
}

\author{
Philip Wikman-Jorgensen ${ }^{1,2}$, Jara Llenas-García ${ }^{1}$, Michael Hobbins $^{3}$, \\ Jochen Ehmer ${ }^{3}$, Rosa Abellana², Alessandra Queiroga Gonçalves ${ }^{2}$, \\ Tomàs Maria Pérez-Porcuna ${ }^{2,4}$ and Carlos Ascaso ${ }^{2}$
}

Affiliations: 'SolidarMed Mozambique, Pemba, Mozambique. ${ }^{2}$ University of Barcelona, Dept of Public Health, Barcelona, Spain. ${ }^{3}$ SolidarMed Switzerland, Lucerne, Switzerland. ${ }^{4}$ Pediathrics department, CAP Valldoreix, Research Unit, Mútua Terrassa Foundation, Mútua Terrassa University Hospital, Terrassa, Spain.

Correspondence: Philip Wikman-Jorgensen, University of Barcelona, Dept of Public Health, Casanova 143, Barcelona 08036, Spain. E-mail: wikman.philipdgmail.com

ABSTRACT The objective of the present study was to assess the diagnostic accuracy of the microscopic observation drug susceptibility (MODS) assay for tuberculosis (TB) diagnosis in HIV-infected patients.

MEDLINE, EMBASE, LILACS, the Cochrane Central Register of Controlled Trials, African Index Medicus, ResearchGate, SciELO, and the abstracts of the main conferences on infectious diseases and tropical medicine were searched, and other sources investigated. Only studies including HIV-infected patients evaluating MODS for the diagnosis of TB and using culture-based diagnostic tests as a gold standard were analysed. Summary sensitivity and specificity were calculated with a bivariate model.

3259 citations were found, 29 were selected for full-text review and 10 studies including 3075 samples were finally analysed. Overall diagnostic accuracy of MODS for the diagnosis of TB was a sensitivity of 88.3\% (95\% CI 86.18-90.2\%) and specificity 98.2\% (95\% CI 97.75-98.55\%). For multidrug-resistant (MDR)-TB, sensitivity was $89 \%$ (95\% CI 66.07-97\%) and specificity was $100 \%$ (95 CI 94.81-100\%). For smear-negative pulmonary TB, a sensitivity of $88.2 \%$ (95\% CI $86.1-89.9 \%)$ and specificity of $98.2 \%(95 \%$ CI $96.8-98.9 \%$ ) were found. Costs varied between USD 0.72 and 7.31 per sample. Mean time to positivity was 8.24 days.

MODS was found to have a good accuracy for the diagnosis of TB and MDR-TB in HIV-infected patients with low cost and fast results.

@ERSpublications

MODS is an accurate, cheap and fast, culture-based technique for diagnosis of TB and MDR-TB in HIV-infected patients http://ow.ly/zEySF

\footnotetext{
This article has supplementary material available from erj.ersjournals.com

Received: April 292014 | Accepted after revision: July 052014 | First published online: Sept 032014

Conflict of interest: None declared.

Copyright @ERS 2014
} 


\section{Introduction}

Tuberculosis (TB) is a global public health priority, being the single leading cause of death by bacterial infection and the second cause of death by an infectious disease [1]. In 2012, there were an estimated 8.6 million new cases of TB (range 8.3-9 million) globally, equivalent to an incidence of 122 cases per 100000 population. TB is the cause of 1.3 million deaths worldwide each year [2]. About 320000 of these deaths are in people living with HIV (PLWH) and it is the first cause of death among PLWH in low-income countries (LICs) [2].

HIV infection increases the risk of having active TB. It has been shown that after seroconversion, the risk of developing $\mathrm{TB}$ doubles and continues to increase as CD4 count declines [3-5]. Moreover, despite immunological recovery with CD4 counts above 500 cells $\mu \mathrm{L}^{-1}$, the risk continues to be two-fold higher than control individuals in the same community [6]. Therefore, the scale-up of antiretroviral treatment and subsequent increase in the prevalence of HIV due to reduced HIV mortality has led to an increase in TB cases in PLWH in some settings $[7,8]$.

The diagnosis of TB in HIV-infected patients is challenging. TB often has an atypical clinical presentation and a paucibacillary nature in PLWH, masking the traditional syndrome of chronic cough, fever and weight loss $[9,10]$. Sputum smear alone fails to diagnose TB in more than $60 \%$ of HIV-infected patients in settings with a high prevalence of HIV infection and does not allow detection of drug resistance [11-13]. Indeed, the difficulties of diagnosing TB in PLWH are among the main factors contributing to the high mortality of HIV/TB co-infection in these settings [14-16]. TB drug resistance is also of special concern in PLWH, due to the extremely high mortality of multidrug-resistant (MDR)-TB and extensively drug resistant (XDR)-TB in these patients [17]. According to the World Health Organization (WHO), in 2012, only 19\% of the estimated incident MDR-TB cases were notified [2].

Improvement and expansion of affordable $\mathrm{TB}$ diagnostic tests is urgently needed to increase the detection of TB, MDR-TB and XDR-TB worldwide [18-20]. New automatic molecular methods such as GeneXpert MTB/ RIF (Cepheid, Sunnyvale, CA, USA) for the diagnosis of TB are currently available [21, 22], but the cost per test is too high for LICs without committed long-term, external funding [23]. In addition, the performance of these tests in HIV-infected patients has been shown to be below what would be desirable [24].

The microscopic observation drug susceptibility (MODS) assay is known as an inexpensive, lowcomplexity, culture-based technique for the diagnosis of TB infection and TB drug resistance [25-27]. It consists in culturing a previously decontaminated sample in liquid culture media and detecting the growth of Mycobacterium microcolonies with an inverted light microscope; direct drug susceptibility testing can be performed at the same time [25]. Previous meta-analyses have shown that MODS performs very well in the diagnosis of TB and MDR-TB in the general population, with a sensitivity of 96\% (95\% CI 94-98\%) and specificity of $96 \%$ (95\% CI $89-100 \%$ ) for the diagnosis of TB, a sensitivity of 98\% (95\% CI 94.5-99.3\%) and specificity of $99.4 \%$ (95\% CI $95.7-99.9 \%)$ for rifampicin resistance, and a sensitivity of $97.7 \%$ (95\% CI $94.4-99.1 \%)$ and specificity of $95.8 \%$ (95\% CI $88.1-98.6 \%)$ for isoniazid resistance $\left(0.1-\mu \mathrm{g} \cdot \mathrm{mL}^{-1}\right.$ cut-off) $[28,29]$. However, they did not specifically analyse PLWH. Although some diagnostic test accuracy studies have included HIV-infected patients, few studies have concluded directly on its accuracy in this target population [30-32]. The potential variation in the performance of MODS in PLWH, and the urgent need for affordable new diagnostic assays for TB and drug-resistant TB in HIV-infected patients led us to perform this study.

The objective of the study was therefore to perform a systematic review and possible meta-analysis of the literature to evaluate the test accuracy of MODS for the diagnosis of active TB and MDR-TB in PLWH. We also aimed to evaluate the diagnostic test accuracy for smear-negative pulmonary TB (SN-PTB) in HIV patients, and to evaluate the time to culture positivity and cost per sample.

\section{Methods}

A specific review protocol was developed and is available in the online supplementary material. Predetermined eligibility criteria to include a study were: assessment of MODS for the diagnosis of active TB with the use of an acceptable reference standard (solid-medium cultures (Löwenstein-Jensen, Middlebrook 7H10 or 7H11, or Ogawa medium) or commercial liquid-culture systems (BACTEC 460TB or BACTEC MGIT 960 Mycobacterial Detection System (BD Biosciences, Franklin Lakes, NJ, USA), BacT/ ALERT (bioMerieux, Lyon, France), or VersaTREK Mycobacteria Detection and Susceptibility (Thermo Scientific, Waltham, MA, USA)) were considered acceptable reference standards); and PLWH had to be included in the population studied, and the data for these patients had to be either directly extractable from the publication or provided by the corresponding authors on request. 
Studies were excluded if: they did not evaluate MODS for the diagnosis of active TB; they did not use an acceptable reference standard; there were no HIV-infected patients included; or the data from the HIVinfected patients was not extractable from the manuscript and the authors did not respond to the data request.

\section{Search strategy and study selection}

The electronic databases MEDLINE, EMBASE, LILACS, the Cochrane Central Register of Controlled Trials, African Index Medicus, ResearchGate and SciELO were screened. Google Scholar and OpenSIGLE were also included as potential resources. The search was performed in March 2013 and updated in June 2014. The abstracts of the main conferences on infectious diseases and tropical medicine held in the years 2011 and 2012 (Interscience Conference on Antimicrobial Agents and Chemotherapy, European Congress on Clinical Microbiology and Infectious Diseases, Annual Meeting of the American Society for Tropical Medicine and Hygiene, European Congress of Tropical Medicine and International Health, Conference on Retroviruses and Opportunistic Infections, and the International AIDS Society Conference) were reviewed, and the references of all the documents recovered were hand-searched for additional studies. The terms "(MODS OR Microscopic Observation Drug Susceptibility) AND tuberculosis" were entered in each database. Neither a restriction in year nor language was considered.

Two authors (P. Wikman-Jorgensen and J. Llenas-García) performed two independent searches and evaluated potential studies of interest according to the inclusion criteria. The publications were screened for potential inclusion by title and abstract, allowing for identification of potentially interesting studies. Eligibility and final inclusion in the review was decided on the basis of a full-text reading. Inclusion in the meta-analysis was also dependent on the availability of the data related to HIV patients and/or the provision of the missing information by the authors through e-mail request. The whole search was documented, including reasons for exclusion. Differences were solved by discussion.

\section{Data collection and analysis}

Two authors (P. Wikman-Jorgensen and J. Llenas-García) independently extracted the data using a standardised data collection form. The data extracted were: full citation; type of study; country of realisation of the study; study setting; date of study execution; reference test; mean and standard deviation of the age of the population included; percentage of females included; presence or absence of symptoms; type and number of sample analysed; mean time to positivity; mean cost per sample; and two-by-two tables of true positives, true negatives, false positives and false negatives. The two-by-two tables were extracted for the diagnosis of TB and MDR-TB in the general (HIV-infected) population and in smear-negative patients.

\section{Assessment of study quality}

The QUADAS-2 criteria were used to evaluate the methodological quality of the studies as recommended by the Cochrane Collaboration [33]. The tool consists of four key domains that judge bias risk and applicability concerns of the studies reviewed by evaluating how patients were selected, the index test, the reference standard, and the flow and timing of samples/patients along the study.

\section{Statistical analysis and data synthesis}

A pooled sensitivity and specificity estimation point was calculated for the diagnosis of TB, MDR-TB and SN-PTB. The bivariate model with the MetaDas Macro was used [34]. Forests plots of sensitivity and specificity as well as the hierarchical summary receiving operation characteristic (HSROC) curves were carried out for each outcome. Data were introduced in Review Manager (version 5.1; The Nordic Cochrane Centre, Copenhagen, Denmark) and later exported to SAS 9.13 (SAS Institute Inc., Cary, NC, USA). To analyse time to positivity and cost per sample, an absolute range was obtained and a sample size weighted mean was planned.

\section{Investigations of heterogeneity and sensitivity analysis}

To investigate causes of heterogeneity of the studies, a subgroup analysis was included in the protocol but was finally not performed due to the small number of studies that met the inclusion criteria. Visual inspection of the forest plots and HSROC curves was used to investigate heterogeneity. A sensitivity analysis was performed to analyse the impact of decisions over the inclusion or exclusion of specific studies.

\section{Results}

The initial screening yielded a total of 3259 citations. Of these, 29 were selected for full-text review and 10 studies were included in the analysis, including a total of 3075 biological samples evaluated for presence of TB bacilli from PLWH (fig. 1) [30-32, 35-41]. 19 out of the 29 initially selected publications were excluded on the basis of: not including $\mathrm{HIV}^{+}$patients [15], insufficient data [2], double publication [1] and not being 


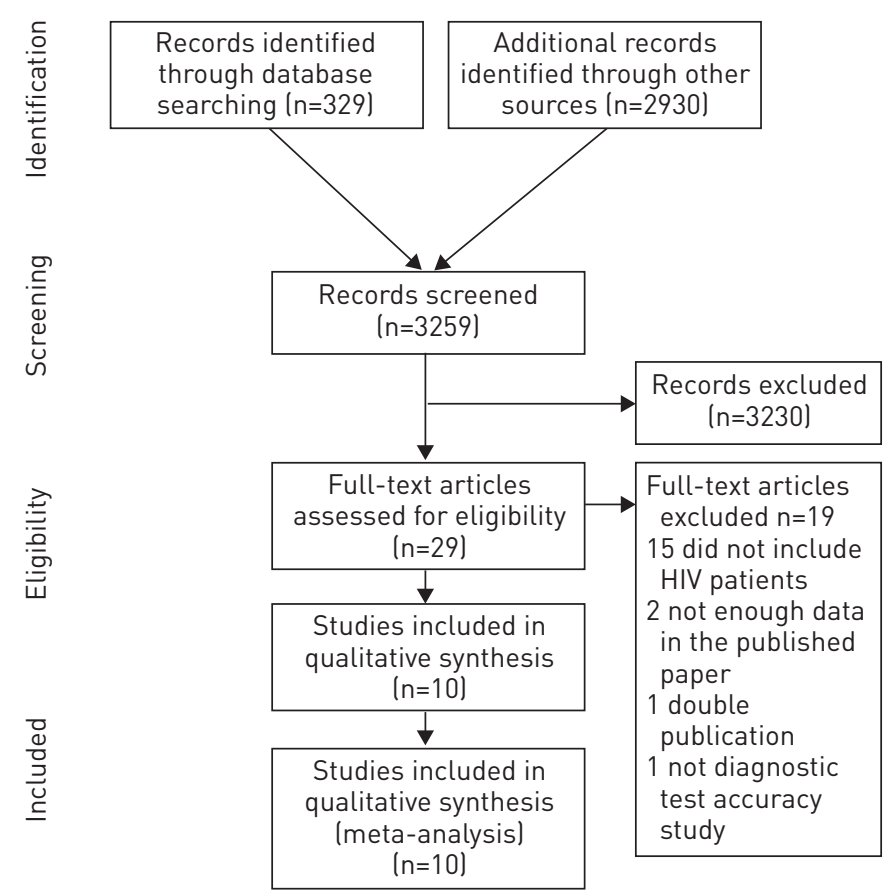

FIGURE 1 Flow diagram of the search and study selection.

a diagnostic accuracy study [1]. The characteristics of the studies included are shown in table 1 . The studies selected were performed on three continents (South America, sub-Saharan Africa and Asia) from 2002 to 2012. Seven studies followed the cross-sectional design [30, 32, 35-38, 40,41] and two were case-control studies [31, 39]. All except one were performed in urban settings [40]. The patients included in the studies varied in race (Afro-American, Asiatic and Hispanic) and age; seven studies included adult patients (mean age 30-35 years) [30, 32, 35-38, 40] and two studies focused on children [36, 39]. A variety of samples were cultured: sputum, induced sputum, string, gastric aspirates, nasopharyngeal aspirates, cerebrospinal fluid (CSF), pleural fluid and stool specimens. One study focused on the diagnosis of TB meningitis [35]; the rest focused on the diagnosis of pulmonary TB. One study was designed to evaluate the performance of the string test versus induced sputum with MODS and Löwenstein-Jensen medium [31]. However, although the data were only partly extractable, the author kindly provided the data and the study was therefore retained in the analysis.

\section{Risk of bias and applicability judgments}

A summary of the risk of bias is shown in table 2 and figure 2. On evaluating the patient selection, in two studies [31,39], it was considered that there was a potentially high risk of bias due to their case-control design. In the index test domain, no study was found to have a high risk of bias. Regarding the reference standard, a potential for a high risk of bias was found in three studies as it is known that the performance of $\mathrm{TB}$ cultures is not optimal for the diagnosis of TB in small children or for the diagnosis of TB meningitis $[35,36,39]$. Regarding flow and timing, two studies showed a high risk of bias as the sampling was not consecutive $[31,32]$. With regard to applicability, we did not find a high degree of concern in any study with respect to patient selection, index test applicability or the reference standard.

\section{Sensitivity and specificity}

The sensitivity varied across studies and the results of each study are summarised in a forest plot (fig. 3a). The lowest sensitivity reported was 77\% (95\% CI 60-90\%) [35] and the highest was 100\% (95\% CI 63-100\%) [31]. The specificity also varied from the lowest 92\% (95\% CI 73-99\%) [37] to the highest 100\% (95\% CI 96-100\%) [31]. The overall sensitivity and specificity found were $88.3 \%$ (95\% CI 86.18-90.19\%) and 98.2\% (95\% CI 97.75-98.55\%), respectively (fig. 4a).

Only four studies reported data on MDR-TB (fig. 3b) [30, 37, 38, 41]. The summary sensitivity and specificity were $89 \%$ (95\% CI 66.07-97\%) and 100\% (95\% CI 94.81-100\%), respectively (fig. 4b).

Data related to the diagnosis of SN-PTB were only retrieved from four studies (fig. 3d) [31, 32, 37, 41]. A summary sensitivity-specificity point was plotted, providing a sensitivity of $88.2 \%$ (95\% CI $86.1-89.9 \%$ ) 


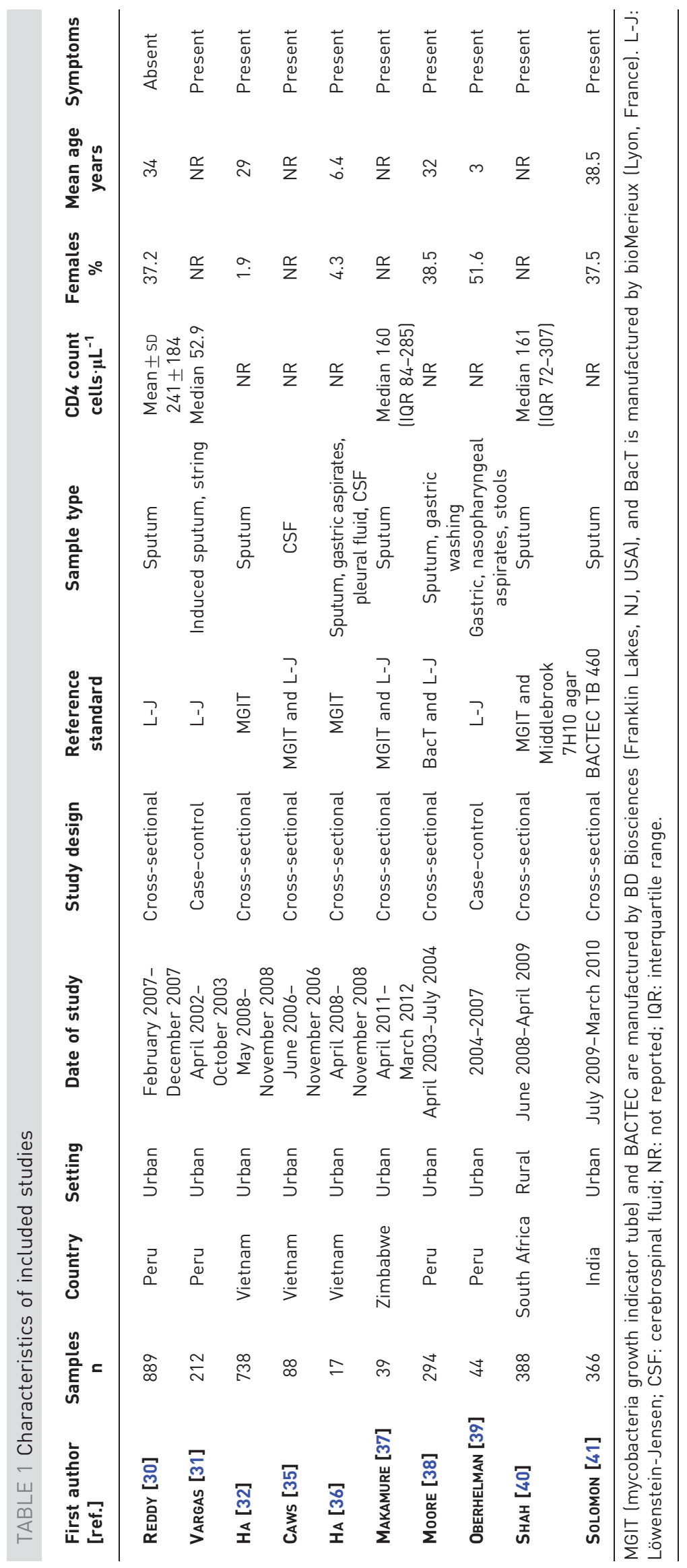


TABLE 2 Risk of bias and applicability concerns summary: review authors' judgements about each domain for each study included

First author [ref.]

Risk of bias

\begin{tabular}{|c|c|c|c|}
\hline $\begin{array}{l}\text { Patient } \\
\text { selection }\end{array}$ & Index test & $\begin{array}{c}\text { Reference } \\
\text { standard }\end{array}$ & $\begin{array}{c}\text { Flow and } \\
\text { timing }\end{array}$ \\
\hline
\end{tabular}

$\begin{array}{cc}+ & + \\ - & +/- \\ + & +/- \\ + & + \\ + & + \\ +/- & + \\ + & + \\ - & +/- \\ + & +/- \\ +/- & +\end{array}$

+
$+/-$
$+/-$
-
-
$+/-$
+
-
$+/-$
+

\begin{tabular}{ccc}
\multicolumn{3}{c}{ Applicability bias } \\
\hline $\begin{array}{c}\text { Patient } \\
\text { selection }\end{array}$ & Index test & $\begin{array}{c}\text { Reference } \\
\text { standard }\end{array}$
\end{tabular}

REdDY [30]
VARGAS [31]
HA [32]
CAWS [35]
HA [36]
MAKAMURE [37]
MOORE [38]
OBERHELMAN [39]
ShAH [40]
SOLOMON [41]

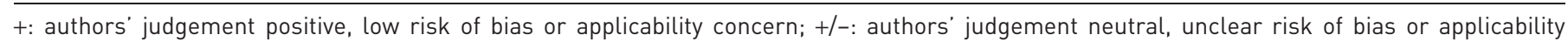
concern; -: authors' judgement negative, high risk of bias or applicability concern.

and a specificity of $98.2 \%$ (95\% CI 96.8-98.9\%). On evaluation by visual inspection of the forest plots, the study of MAKAMURE et al. [37] was found to be an outlier. A sensitivity analysis was performed excluding this study, and a sensitivity of $88.8 \%$ (95\% CI $84.1-92.3 \%$ ) and a specificity of $98.2 \%$ (95\% CI 97.7-98.6\%) were found.

CAws et al. [35] evaluated the sensitivity and specificity of MODS for the diagnosis of TB meningitis. They showed a sensitivity of $77 \%(95 \%$ CI $60-90 \%)$ and a specificity of $100 \%$ (95\% CI 93-100\%) when compared with other cultures (MGIT and Löwenstein-Jensen). On assessing the forest plots and the HSROC curves for heterogeneity, this study was found to be an outlier, as it showed a sensitivity somewhat below the remaining studies. A sensitivity analysis was performed excluding this study and the summary sensitivity increased to $95 \%$ (95\% CI 95-96\%).

Two studies were found to evaluate the usefulness of MODS in HIV-infected children [36, 39]. The samples used were heterogeneous (sputum, gastric fluid, nasopharyngeal aspirates, pleural fluid, CSF and stools) and were presented as a pooled analysis in the original studies. The sensitivity in children living with HIV was $80 \%(95 \%$ CI $28-99 \%)$ in one study and $100 \%(95 \%$ CI $3-100 \%)$ in the other $[36,39]$. In one of these studies, sputum samples were used and sensitivity was $80 \%$ (95\% CI 28-100\%) [36]. The specificity was high in both studies, being 100\% (95\% CI 74-100\%) and 100\% (95\% CI 92-100\%). A sensitivity analysis was performed excluding these studies and the pooled estimate remained almost unaltered (sensitivity 88.1\% (95\% CI 83-91.8\%) and specificity 98.2 (95\% CI 98.1-98.3\%)).

Data about the time to culture positivity was only retrieved in five studies [30, 32, 36, 37, 41], varying from study to study and ranging from 5.5 to 14.3 days. As individual-level patient data were not available, a mean weighted by the study sample size was calculated, yielding a result of 8.24 days till culture positivity.

Information about the cost per sample processed for $\mathrm{TB}$ diagnosis was only found in four studies [30, 32, 35, 38]. CAws et al. [35], HA et al. [32] and Moore et al. [38] only reported costs for reagents and consumables, and did not account for labour, capital or overhead costs. A sample size weighted mean of the cost per sample was USD 0.72 per test (absolute range USD 0.53-2.00). REDDY et al. [30] took a more comprehensive approach, obtaining a total cost per sample of USD 7.31.

\section{Discussion}

To our knowledge this is the first systematic review and meta-analysis to evaluate the performance of MODS in PLWH. The results suggest that MODS could be a valuable technique for the diagnosis of TB in PLWH, with good sensitivity and specificity, allowing for faster results than conventional culture techniques and at a lower cost.

MODS was also found to have both a good sensitivity and specificity for the diagnosis of active MDR-TB. Although the number of studies included was small and results should be interpreted with caution, these results are in line with those published on the diagnosis of MDR-TB by MODS in the general population 
FIGURE 2 Risk of bias and applicability concerns: review authors' judgements about each domain across the 10 studies included.

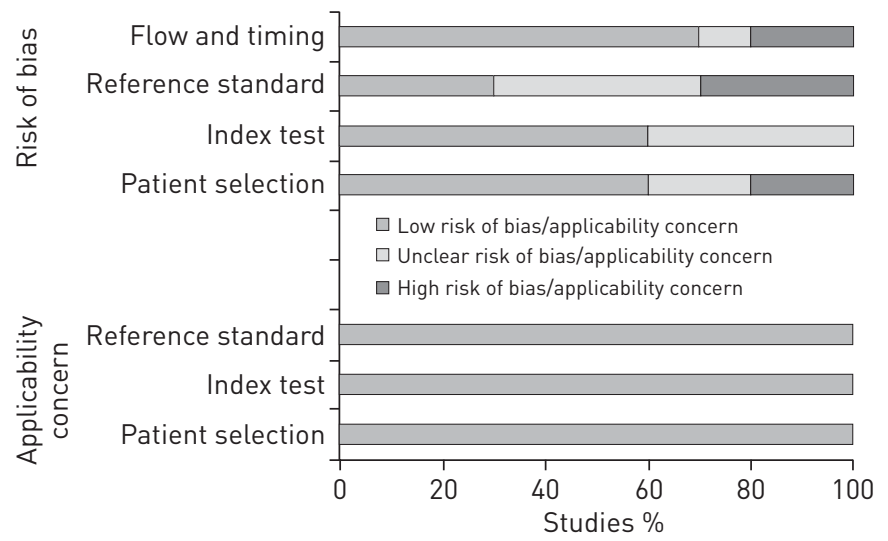

(sensitivity $97.7 \%$ and specificity $95.8 \%$ for isoniazid resistance, and sensitivity $98 \%$ and specificity $98.6 \%$ for rifampicin resistance) [29]. Being able to obtain the results of drug susceptibility testing within 8 days and the possibility of also performing second-line drug susceptibility testing make MODS a very interesting and attractive technique for LICs [42, 43]. Moreover, it has recently been validated for DST in patients failing first-line TB treatment [44].

Due to the paucibacillary nature of TB in HIV-infected patients, the performance of MODS in SN-PTB is especially interesting. A summary sensitivity of $88.2 \%$ (95\% CI $86.1-89.9 \%)$ and a specificity of $98.2 \%(95 \%$ CI 96.8-98.9\%) for sputum cultures in SN-PTB was found. These results are in line with another recently published study that evaluated the performance of MODS in SN-PTB [45]. Moreover, data from the study by VARGAS et al. [31] suggest that if a string test is used in smear-negative HIV-infected patients, the sensitivity and specificity of MODS are higher than those of standard microbiological cultures.

Another important issue in PLWH is how MODS performs in the diagnosis of extrapulmonary TB and in samples other than sputum. Data are again scarce and the different samples used in the different studies did not allow stratified analysis. The only extrapulmonary form of TB that we could evaluate was TB meningitis, and the results showed that MODS appears to be a promising technique with a diagnostic yield in line with other culture techniques [46]. However, the sensitivity was lower than that for sputum cultures. The authors of the study suggested that the volume cultured played a role in this low sensitivity, as they used $100 \mu \mathrm{L}$ of deposit for MODS and $250 \mu \mathrm{L}$ for Löwenstein-Jensen and MGIT. In a previous study, the same group found that the volume of CSF had a considerable influence on the positivity rate of the cultures [47]. Moreover, our sensitivity analysis showed that this study had a clear impact on the pooled estimates, suggesting that the sample type influences the sensitivity and specificity of the technique. Further studies are needed to evaluate how to optimise MODS in the diagnosis of TB meningitis and other forms of extrapulmonary TB in PLWH.

All the studies were designed to assess the performance of MODS in symptomatic patients but that of REDDY et al. [30] addressed its performance as a screening tool for asymptomatic HIV-infected patients. This specific use of MODS is interesting as ruling out TB in PLWH before starting isoniazid preventive therapy (IPT) is challenging in clinical practice. There is an ongoing debate about the best screening method for pulmonary TB among PLWH in LICs [11]. MODS may have an important role as it delivers results faster than other mycobacterial cultures. Nevertheless, its complexity may be a drawback. In their study, REDDY et al. [30] proposed that liquid culture of two sputum samples alone can be used as an effective screening strategy for pulmonary TB before IPT in PLWH. More research on the use of MODS as a screening strategy, alone or in combination with other screening strategies, in LICs is needed as IPT has the potential to save millions of lives and provide an important contribution to the control of TB in regions with a high burden of HIV infection.

The diagnosis of TB in children is challenging. Two studies have analysed the performance of MODS in HIV-infected children, showing a sensitivity and specificity that were roughly the same as for other mycobacterial cultures. However, it is known that in the case of children, the diagnosis of TB is difficult due to the paucibacillary nature of this infection in this population, and cultures do not diagnose all cases of active TB [48]. A sensitivity analysis was performed excluding the studies evaluating children, as doubts arose whether to include them or not, as no age restriction had been pre-specified in the study protocol. Nonetheless, the pooled estimate remained unaltered. 
a)

First author [ref.]
CAWs [35]
Ha [36]
Ha [32]
MAKAMURe [37]
SHAH [40]
Solomon [41]
Reddy [30]
MoORe [38)
VARgAs [31]a
VARgAs [31]b
OberheLMAn [39]

$\begin{array}{cccc}\text { TP } & \text { FP } & \text { FN } & \text { TN } \\ 27 & 0 & 8 & 53 \\ 4 & 0 & 1 & 12 \\ 431 & 6 & 101 & 200 \\ 11 & 2 & 2 & 22 \\ 74 & 9 & 10 & 295 \\ 83 & 8 & 10 & 265 \\ 30 & 12 & 2 & 845 \\ 43 & 3 & 1 & 247 \\ 8 & 1 & 0 & 271 \\ 11 & 3 & 0 & 266 \\ 1 & 0 & 0 & 43\end{array}$

Se $(95 \% \mathrm{CI})$

$0.77(0.60-0.90)$

$0.80(0.28-0.99)$

$0.81(0.77-0.84)$

$0.85(0.55-0.98)$

$0.88(0.79-0.94)$

0.89 (0.81-0.95)

0.94 (0.79-0.99)

$0.98(0.88-1.00)$

$1.00(0.63-1.00)$

$1.00(0.72-1.00)$

1.00 (0.03-1.00)
Sp $(95 \% \mathrm{CI})$

$1.00(0.93-1.00)$

$1.00(0.74-1.00)$

$0.97(0.94-0.99)$

$0.92(0.73-0.99)$

$0.97(0.94-0.99)$

$0.97(0.94-0.99)$

$0.99(0.98-0.99)$

$0.99(0.97-1.00)$

$1.00(0.98-1.00)$

$0.99(0.97-1.00)$

$1.00(0.92-1.00)$

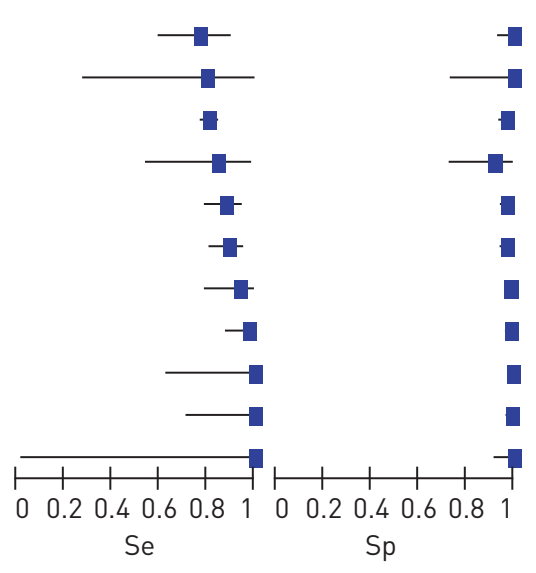

$\mathrm{Sp}(95 \% \mathrm{CI})$

0.75 (0.19-0.99)

$1.00(0.95-1.00)$

$1.00(0.92-1.00)$

$1.00(0.99-1.00)$

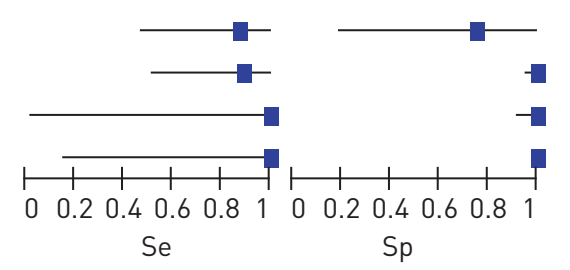

Sp (95\% CI)

$1.00(0.54-1.00)$

$0.97(0.94-0.99)$

$0.92(0.73-0.99)$

$0.97(0.94-0.99)$

$0.97(0.94-0.99)$

$0.99(0.98-0.99)$

$0.99(0.97-1.00)$

$1.00(0.98-1.00)$

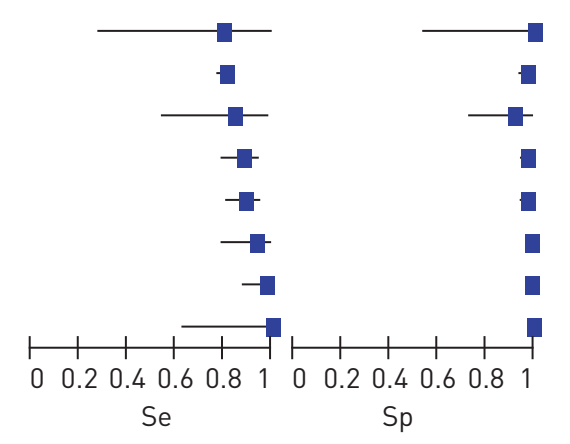

Sp $(95 \%$ CI)

$0.91(0.72-0.99)$

$0.91(0.82-0.97)$

0.97 (0.94-0.99)

$1.00(0.98-1.00)$

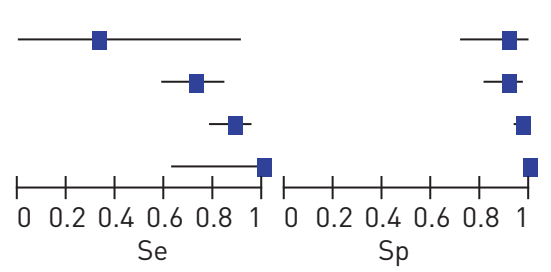

FIGURE 3 Forest plot of sensitivity (Se) and specificity (Sp) of microscopic observation drug susceptibility assays for the diagnosis of a) tuberculosis (TB), b) multidrug-resistant TB, c) pulmonary TB and d) smear-negative pulmonary TB, in people living with HIV. VARGAS [31] a and b are results for induced sputum and string, respectively. TP: true positive, FP: false positive; FN: false negative; TN: true negative.

A high degree of heterogeneity of how costs were counted was found. Only counting costs for reagents and consumables, the cost varied from USD 0.53 to USD 2.00 per test. When a more comprehensive approach was used, the costs rose to USD 7.31 per test. This is very inexpensive in comparison with other alternatives, such as automated liquid cultures (average cost USD 16-32 per test) [49], or even subventioned rapid molecular assays, such as GeneXpert MTB/RIF (cost per cartridge USD 9.98 ex works) [50-52]. Nevertheless, capital, labour, overhead and other costs also have to be accounted for. Pragmatic trials in LICs should be encouraged to choose the best diagnostic option for these patients in these settings, evaluating not only diagnostic test accuracy but also its cost-effectiveness and affordability. 
a)
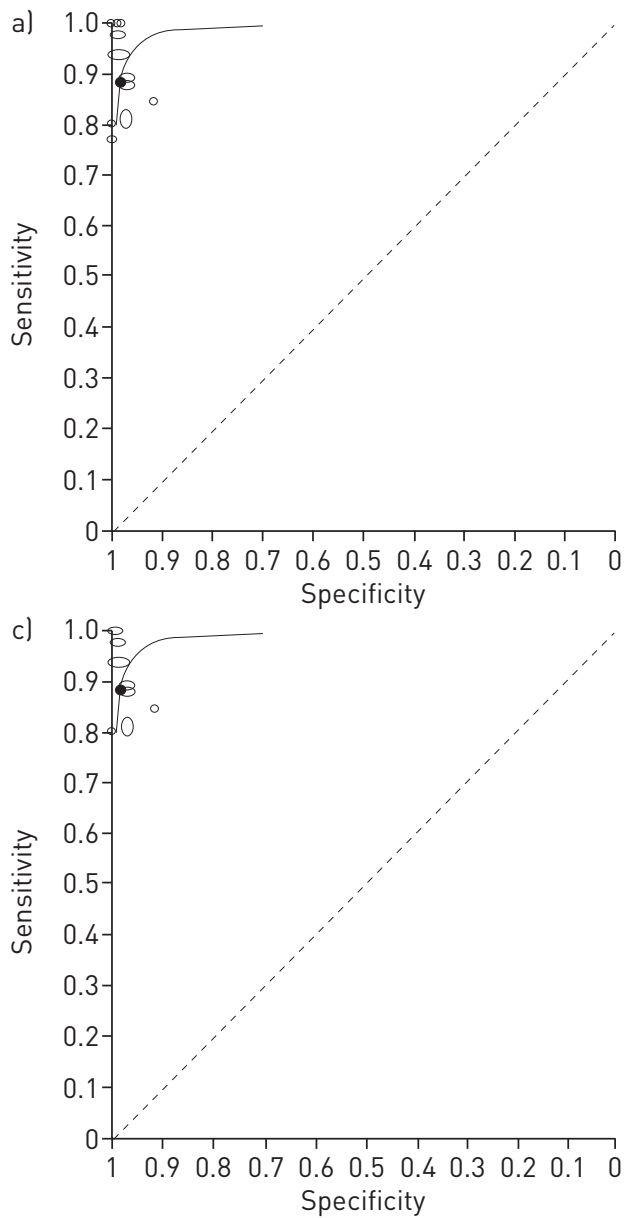
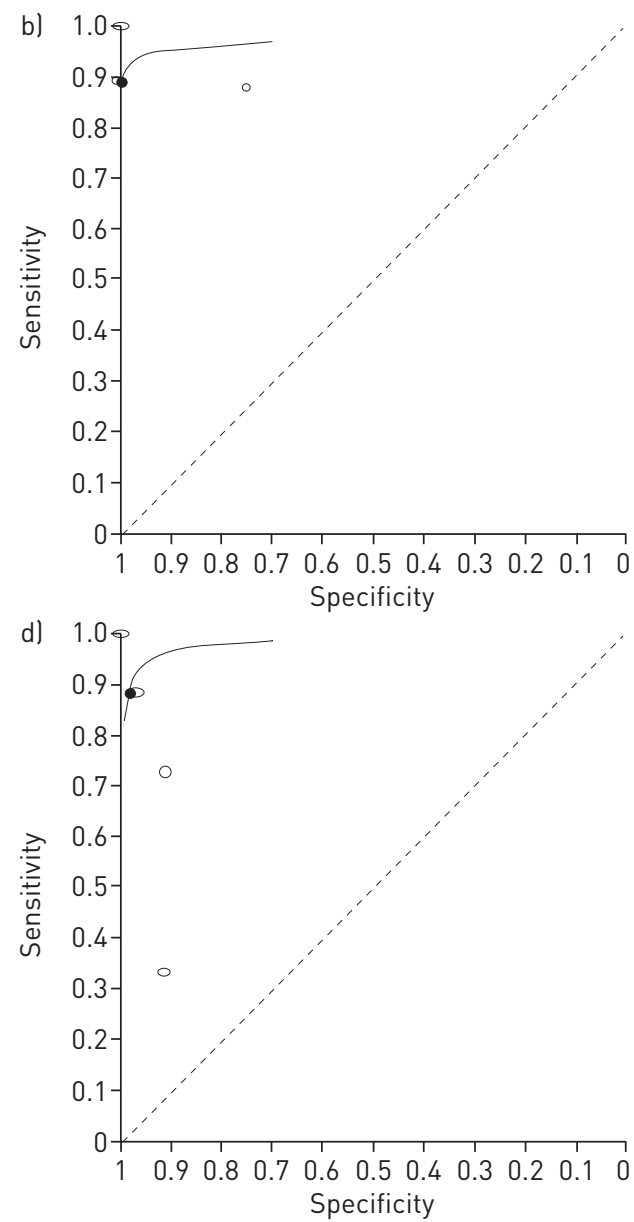

FIGURE 4 Hierarchichal summary receiving operating curves of microscopic observation drug susceptibility assays for the diagnosis of a) active tuberculosis (TB), b) multidrug-resistant TB, c) pulmonary TB and d) smear-negative pulmonary TB, in people living with HIV. Open circles represent individual studies, with the size of the circle proportionate to the study. The summary point is a closed circle, representing sensitivity and specificity estimates pooled with a bivariate random-effects model.

The greatest limitation of this study is the small number of studies included, as the number of studies evaluating MODS in PLWH is low. Moreover, many studies assessing the performance of MODS performed in settings with a high prevalence of HIV infection do not report separate data for PLWH and, in some of them, HIV testing was not systematically performed in TB patients [53-55]. Only four of the 10 studies included were specifically designed to address the performance of MODS in PLWH [30-32, 39]. Nevertheless, the search for published studies was thorough: a broad search in electronic medical databases as well as grey literature databases, and conference books of abstracts were also searched for studies. In addition, the authors of studies that did not provide complete data in the published papers were contacted to provide missing data. Further studies to assess the performance of MODS in HIV-infected adults and children in LICs are needed and disaggregation of data of PLWH should be encouraged. Another limitation is that quite a high risk of bias was found on assessing the quality of the studies included, being mainly related to the reference standard used. Cultures were not considered to correctly classify the target condition in many studies, reflecting the fact that there is no true gold standard for the diagnosis of TB. However, TB cultures are believed to be the best proxy available.

MODS was specifically developed and designed to be set up in LICs. Nevertheless, there are concerns about its feasibility in rural areas, and WHO has not recommended the implementation of MODS in rural laboratories at district levels but has recommended the use of the GeneXpert MTB/RIF assay [56]. The development of the GeneXpert MTB/RIF assay is undoubtedly a landmark event. It is easy to use and fast; in a recent meta-analysis by the Cochrane Collaboration Group, it showed a pooled sensitivity in PLWH of 80\% (95\% CI 67-88\%); and it has a low biohazard level [24]. In modelling studies, cost-effectiveness has 
been evaluated and it has been postulated as cost-effective $[57,58]$. However, it has been deemed to be too expensive for a point-of-treatment setting and has been recommended to be installed only at laboratory facilities [59]. In addition, clinical and programmatic effects have recently been evaluated; results have not been so encouraging since the inclusion of more patients on same-day treatment and more culture-positive patients on treatment; and shorter time to treatment did not translate into lower TB morbidity [60]. This study also suggested that the cost-effectiveness may have been overestimated. However, MODS may perform better in PLWH and could be less costly. Studies directly comparing MODS and GeneXpert MTB/ RIF, especially in high HIV burden settings, could help defining better diagnostic strategies for TB and MDR-TB in LICs.

In summary, we found MODS to be a good technique for the diagnosis of TB, MDR-TB and SN-PTB in PLWH. However, the data available are scarce, and more studies on its performance in HIV-infected patients and comparisons with molecular techniques such as GeneXpert MTB/RIF should be encouraged.

\section{Acknowledgements}

We would like to thank all the investigators who contributed with data after the request from the authors, namely Dang Thi Minh Ha (Pham Ngoc Thach Hospital, Ho Chi Minh City, Vietnam), John Metcalfe (Francis J. Curry International Tuberculosis Center, Division of Pulmonary and Critical Care Medicine, San Francisco General Hospital, University of California, San Francisco, CA, USA), Daniel Vargas (Universidad Peruana Cayetano Heredia, Lima, Peru), Suniti Solomon, Ramachandran Vignesh (both Infectious Diseases Laboratory, YRG Centre for AIDS Research and Education, Chennai, India) and Richard Oberhelman (Tulane School of Public Health and Tropical Medicine, New Orleans, LA, USA). We would also like to thank David Moore (Hospital for Tropical Diseases, London, UK) for his kind support.

\section{References}

Russell DG, Nathan CF. Graduation time. Nature 2013; 502: S7.

WHO. Global Tuberculosis Report 2013. www.who.int/tb/publications/global_report/en/.

Sonnenberg P, Glynn JR, Fielding K, et al. How soon after infection with HIV does the risk of tuberculosis start to increase? A retrospective cohort study in South African gold miners. J Infect Dis 2005; 191: 150-158.

4 Holmes CB, Wood R, Badri M, et al. CD4 decline and incidence of opportunistic infections in Cape Town, South Africa: implications for prophylaxis and treatment. J Acquir Immune Defic Syndr 2006; 42: 464-469.

5 Glynn JR, Murray J, Bester A, et al. Effects of duration of HIV infection and secondary tuberculosis transmission on tuberculosis incidence in the South African gold mines. AIDS 2008; 22: 1859-1867.

6 Lawn SD, Myer L, Edwards D, et al. Short-term and long-term risk of tuberculosis associated with CD4 cell recovery during antiretroviral therapy in South Africa. AIDS 2009; 23: 1717-1725.

7 Auld AF, Mbofana F, Shiraishi RW, et al. Incidence and determinants of tuberculosis among adults initiating antiretroviral therapy - Mozambique, 2004-2008. PLoS One 2013; 8: e54665.

8 Zaidi J, Grapsa E, Tanser F, et al. Dramatic increase in HIV prevalence after scale-up of antiretroviral treatment. AIDS 2013; 27: 2301-2305.

9 Sterling TR, Pham PA, Chaisson RE. HIV infection-related tuberculosis: clinical manifestations and treatment. Clin Infect Dis 2010; 50: Suppl. 3, S223-S230.

10 Lee MP, Chan JWM, Ng KKP, et al. Clinical manifestations of tuberculosis in HIV-infected patients. Respirology 2000; 5: 423-426.

11 Reid MJ, Shah NS. Approaches to tuberculosis screening and diagnosis in people with HIV in resource-limited settings. Lancet Infect Dis 2009; 9: 173-184.

12 Getahun H, Harrington M, O’Brien R, et al. Diagnosis of smear-negative pulmonary tuberculosis in people with HIV infection or AIDS in resource-constrained settings: informing urgent policy changes. Lancet 2007; 369 : 2042-2049.

13 Siddiqi K, Lambert ML, Walley J. Clinical diagnosis of smear-negative pulmonary tuberculosis in low-income countries: the current evidence. Lancet Infect Dis 2003; 3: 288-296.

14 Gandhi NR, Shah NS, Andrews JR, et al. HIV coinfection in multidrug- and extensively drug-resistant tuberculosis results in high early mortality. Am J Respir Crit Care Med 2010; 181: 80-86.

15 Cain KP, Anekthananon T, Burapat C, et al. Causes of Death in HIV-infected Persons Who Have Tuberculosis, Thailand. Emerg Infect Dis 2009; 15: 258-264.

16 Mukadi YD, Maher D, Harries A. Tuberculosis case fatality rates in high HIV prevalence populations in subSaharan Africa. AIDS 2001; 15: 143-152.

17 Gandhi NR, Moll A, Sturm AW, et al. Extensively drug-resistant tuberculosis as a cause of death in patients coinfected with tuberculosis and HIV in a rural area of South Africa. Lancet 2006; 368: 1575-1580.

18 Vignesh R, Balakrishnan P, Shankar EM, et al. High rate of acquired resistance to tuberculosis among HIV-infected subjects of Chennai, South India. J Infect 2007; 55: e141-e142.

19 Dheda K, Gumbo T, Gandhi NR, et al. Global control of tuberculosis: from extensively drug-resistant to untreatable tuberculosis. Lancet Respir Med 2014; 2: 321-338.

20 Perkins MD, Cunningham J. Facing the crisis: improving the diagnosis of tuberculosis in the HIV era. J Infect Dis 2007; 196: Suppl., S15-S27.

21 Lawn SD, Mwaba P, Bates M, et al. Advances in tuberculosis diagnostics: the Xpert MTB/RIF assay and future prospects for a point-of-care test. Lancet Infect Dis 2013; 13: 349-361.

22 Weyer K, Mirzayev F, Migliori GB, et al. Rapid molecular TB diagnosis: evidence, policy making and global implementation of Xpert MTB/RIF. Eur Respir J 2013; 42: 252-271.

23 Pantoja A, Fitzpatrick C, Vassall A, et al. Xpert MTB/RIF for diagnosis of tuberculosis and drug-resistant tuberculosis: a cost and affordability analysis. Eur Respir J 2013; 42: 708-720. 
24 Steingart K, Sohn H, Schiller I, et al. Xpert $\circledast^{3}$ MTB/RIF assay for pulmonary tuberculosis and rifampicin resistance in adults. Cochrane Database Syst Rev 2013; 1: CD009593.

25 Caviedes L, Lee T, Gilman R. Rapid, efficient detection and drug susceptibility testing of Mycobacterium tuberculosis in sputum by microscopic observation of broth cultures. J Clin Microbiol 2000; 38: 1203-1208.

26 Mendoza A, Castillo E, Gamarra N, et al. Reliability of the MODS assay decentralisation process in three health regions in Peru. Int J Tuberc Lung Dis 2011; 15: 1-12.

27 Moore DAJ, Mendoza D, Gilman RH, et al. Microscopic observation drug susceptibility assay, a rapid, reliable diagnostic test for multidrug-resistant tuberculosis suitable for use in resource-poor settings. J Clin Microbiol 2004; 42: 4432-4437.

28 Leung E, Minion J, Benedetti A, et al. Microcolony culture techniques for tuberculosis diagnosis: a systematic review. Int J Tuberc lung Dis 2011; 16: 16-23.

29 Minion J, Leung E, Menzies D, et al. Microscopic-observation drug susceptibility and thin layer agar assays for the detection of drug resistant tuberculosis: a systematic review and meta-analysis. Lancet Infect Dis 2010; 10: 688-698.

30 Reddy KP, Brady MF, Gilman RH, et al. Microscopic observation drug susceptibility assay for tuberculosis screening before isoniazid preventive therapy in HIV-infected persons. Clin Infect Dis 2010; 50: 988-996.

31 Vargas D, García L, Gilman RH, et al. Diagnosis of sputum-scarce HIV-associated pulmonary tuberculosis in Lima, Peru. Lancet 2005; 365: 150-152.

32 Ha DTM, Lan NTN, Kiet VS, et al. Diagnosis of pulmonary tuberculosis in HIV-positive patients by microscopic observation drug susceptibility assay. J Clin Microbiol 2010; 48: 4573-4579.

33 Whiting P, Rutjes AWS, Reitsma JB, et al. The development of QUADAS: a tool for the quality assessment of studies of diagnostic accuracy included in systematic reviews. BMC Med Res Methodol 2003; 3: 25.

34 Takwoingi Y, Deeks J. METADAS: a SAS macro for meta-analysis of diagnostic accuracy studies. http://srdta. cochrane.org/sites/srdta.cochrane.org/files/uploads/METADAS Readme v1.3.pdf.

35 Caws M, Dang TMH, Torok E, et al. Evaluation of the MODS culture technique for the diagnosis of tuberculous meningitis. PLoS One 2007; 2: el173.

36 Ha DTM, Lan NTN, Wolbers M, et al. Microscopic observation drug susceptibility assay (MODS) for early diagnosis of tuberculosis in children. PLoS One 2009; 4: e8341.

37 Makamure B, Mhaka J, Makumbirofa S. Microscopic-observation drug-susceptibility assay for the diagnosis of drug-resistant tuberculosis in Harare, Zimbabwe. PLoS One 2013; 8: 1-7.

38 Moore DA, Evans CA, Gilman RH, et al. Microscopic-observation drug-susceptibility assay for the diagnosis of TB. N Engl J Med 2006; 355: 1539-1550.

39 Oberhelman RA, Soto-Castellares G, Gilman RH, et al. Diagnostic approaches for pediatric tuberculosis among HIV-infected and HIV-uninfected children in Peru. Am J Trop Med Hyg 2012; 87: Suppl., 423.

40 Shah NS, Moodley P, Babaria P, et al. Rapid diagnosis of tuberculosis and multidrug resistance by the microscopicobservation drug-susceptibility assay. Am J Respir Crit Care Med 2011; 183: 1427-1433.

41 Solomon S, Balakrishnan P, Vignesh R, et al. A rapid and low-cost microscopic observation drug susceptibility assay for detecting TB and MDR-TB among individuals infected by HIV in South India. Ind J Med Microbiol 2013; 31: 130-137.

42 Fitzwater SP, Sechler GA, Jave O, et al. Second-line anti-tuberculosis drug concentrations for susceptibility testing in the MODS assay. Eur Respir J 2013; 41: 1163-1171.

43 Trollip A, Moore D, Coronel J, et al. Second-line drug susceptibility breakpoints for Mycobacterium tuberculosis using the MODS assay. Int J Tuberc Lung Dis 2014; 18: 227-232.

44 Coronel J, Roper M. Validation of microscopic observation drug susceptibility testing for rapid, direct rifampicin and isoniazid drug susceptibility testing in patients receiving tuberculosis. Clin Microbiol Infect 2014; 20: 536-541.

45 Walusimbi S, Bwanga F, De Costa A, et al. Meta-analysis to compare the accuracy of GeneXpert, MODS and the WHO 2007 algorithm for diagnosis of smear-negative pulmonary tuberculosis. BMC Infect Dis 2013; 13: 507.

46 Rock RB, Olin M, Baker CA, et al. Central nervous system tuberculosis: pathogenesis and clinical aspects. Clin Microbiol Rev 2008; 21: 243-261.

47 Thwaites G, Chau T, Farrar J. Improving the bacteriological diagnosis of tuberculous meningitis. J Clin Microbiol 2004; 42: 378-379.

48 Pérez-Porcuna TM, Ascaso C, Ogusku MM, et al. Evaluation of new strategies for the diagnosis of tuberculosis among pediatric contacts of tuberculosis patients. Pediatr Infect Dis J 2012; 31: e141-e146.

49 Dowdy DW, Steingart KR, Pai M. Serological testing versus other strategies for diagnosis of active tuberculosis in India: a cost-effectiveness analysis. PLoS Med 2011; 8: e1001074.

50 UNITAID. UNITAID approves US\$ 30 million for innovaative project to roll out ground-breaking tuberculosis test at reduced cost. www.unitaid.eu/resources/news/releases/943-unitaid-approves-us-30-million-for-innovative-project-to-roll-out-ground-breaking-tuberculosis-test-at-reduced-cost Date last accessed: July 29, 2013. Date last updated: June 13, 2013.

51 Bill and Melinda Gates Foundation. Public-private partnership announces immediate 40 percent cost reduction for rapid TB test. www.gatesfoundation.org/press-releases/Pages/public-private-partnership-40-percent-reduction-TBtest.aspx Date last accessed: July 29, 2013. Date last updated: August 6, 2012.

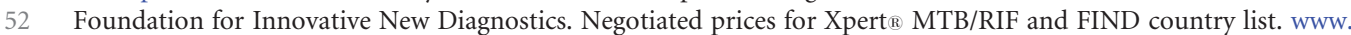
finddiagnostics.org/about/what_we_do/successes/find-negotiated-prices/xpert_mtb_rif.html Date last accessed: July 29, 2013. Date last updated: October 2013.

53 Arias M, Mello FCQ, Pavón A, et al. Clinical evaluation of the microscopic-observation drug-susceptibility assay for detection of tuberculosis. Clin Infect Dis 2007; 44: 674-680.

54 Michael JS, Daley P, Kalaiselvan S, et al. Diagnostic accuracy of the microscopic observation drug susceptibility assay: a pilot study from India. Int J Tuberc Lung Dis 2010; 14: 482-488.

55 Bwanga F, Haile M, Joloba ML, et al. Direct nitrate reductase assay versus microscopic observation drug susceptibility test for rapid detection of MDR-TB in Uganda. PLoS One 2011; 6: e19565. 
56 World Health Organization. Policy framework for implementing new tuberculosis diagnostics. www.who.int/ tb/laboratory/whopolicyframework_rev_june2011.pdf Date last accessed: October 25, 2013. Date last updated: March 2010.

57 Vassall A, van Kampen S, Sohn H, et al. Rapid diagnosis of tuberculosis with the Xpert MTB/RIF assay in high burden countries: a cost-effectiveness analysis. PLoS Med 2011; 8: e1001120.

58 Andrews J, Lawn S, Rusu C, et al. The cost-effectiveness of routine tuberculosis screening with Xpert MTB/RIF prior to initiation of antiretroviral therapy in South Africa: a model-based analysis. AIDS 2012; 26: 987-995.

59 Schnippel K, Meyer-Rath G, Long L, et al. Scaling up Xpert MTB/RIF technology: the costs of laboratory- vs. clinicbased roll-out in South Africa. Trop Med Int Health 2012; 17: 1142-1151.

60 Theron G, Zijenah L, Chanda D, et al. Feasibility, accuracy, and clinical effect of point-of-care Xpert MTB/RIF testing for tuberculosis in primary-care settings in Africa: a multicentre, randomised, controlled trial. Lancet 2013; 6736: 1-12. 